\title{
A Review: Smart Ambulance and Traffic Controlling System
}

\author{
Sudhakara H M ${ }^{1}$, Girish H. R ${ }^{2}$, Kumara Swamy N. R ${ }^{3}$, J. Vinay Kumar ${ }^{4}$ and Sachin Kumar. M $^{5}$ \\ ${ }^{1}$ Sr. Assistant Professor, ECE, AIET, Mangalore, India \\ ${ }^{2345}$ UG Scholar, Dept of ECE, AIET, Mangalore, India
}

\begin{abstract}
India is a developing country, population of India is significantly growing. India stands within the 2nd place in the world in terms of population. As there will be increase in population gradually there will be increase in number of vehicles, due to which the traffic congestion increases and because of which the emergency vehicles like ambulance, fire engine etc. face difficult to reach the destination in time. Under these circumstances, a promising system that can clear the traffic signal especially $i$ $n$ peak hours and thus provide a safe route for emergency vehicles is extremely important. In existing literature there's less focus show on the emergency vehicles to clear the trail, to overcome this issue a RFID based system is proposed by using this technique we will manage and regulate the traffic signals at junction which emergency vehicle approaches. Thus there'll be easy passing out for the emergency vehicles in traffic congestion. The proposed frame work is modeled by the means of an experimental setup using Arduino and LED displays which simulates a true time traffic scenario. This simulation results illustrate the terms of detection still as is providing passing for the emergency vehicle to of holdup in peak hours.
\end{abstract}

Keywords:-RFID technology, traffic signal management, congestion clearance;

\section{INTRODUTION}

Population in developing countries such as India is increasing significantly. This result in a number of problems such as heavy traffic jams, violation of the traffic rules and sometimes even accidents. For example, the number of road accidents in major cities s uch as Chennai, Hyderabad and Delhi increased to 16 death s per hour,as stated by the Indian Government.

Additionally,traffic congestion leads to long waiting times, $\mathrm{f}$ uel depletion and even money waste.

In particular, traffic congestion contributes to high rates of e missions impacting the health of the local population, shuttl es and animals. Traffic congestion is often commonlyassocia ted with some other traffic issues, such as the blocking ofe mergency vehicles. Precisely, the traffic congestion often blocks the path of the emergency vehicles which may Human Life is a very valuable thing for any country. The regular occurrence of incidents and medical emergencies su ch

fire, road accidents, medical emergencies etc. It is very nece ssary that emergency vehicles arrive on time to prevent seri ous loss of humans. Thus, hospitals and fire stations are throughout the city to reduce response time in case of such emergencies.

A very rapid population growth in cities has resulted in trem endous road traffic within the city.In addition, in recent time $s$ the number of deaths due to delays in the arrival of emerg ency vehicle

has risen to greater extent.Hence emergency services such a $s$ ambulances and fire engines must be on time to avoid loss of human life.In the current traffic situation, therefore, help ing an emergency vehicle move out of traffic congestion is very much important. To solve the problems given above. In this paper, we have come up with the 'Smart Ambulance and traffic controlling system'. The main purpose of this device is to allow the ambulance to reach a specific location without making it sto p somewhere before it reaches the destination.

\section{LITERATURE SURVEY}

Vanjale et al [1] have proposed a RFID-based system, which manages and regulates the traffic signals at junctions when the emergency vehicle approaches, by allowing the straight forward passage out of the traffic congestions. This paper proposes an approach which controls the Traffic Signals so as that when the emergency vehicle is on its way to a selected destination. The case of ambulance is tracked by using GPS. This location is send to the application. The application performs the algorithm with the help of this data and so the google map. It controls the signals on its path. They also introduced a current blue light to stoplight to avoid the chaos within the mind of the people waiting at the stoplight. The working of the system relies on two important modules.

\section{- $\quad$ The GPS System \\ - Application Server}

The ambulance or any emergency vehicle must be equipped with the GPS System. This GPS System will send the coordinates of the vehicle at every moment to application Server. Each vehicle must be logged in to the android application. This application keeps tracking the vehicle and tracks the route. The route is already selected by the motive force this route is additionally accessed by the server. The applying server accepts all the knowledge, based on this information the server finds this location of the vehicle and also the route selected to the destination. This helps to look out the following stoplight in its vicinity. Whenever the vehicle comes within the space of certain meters from the signal the server must send the required action so as that the vehicle doesn't must wait at the signal. A symbol is additionally sent to the destination hospital so as that the hospital authorities are able to handle the patient. Hospitals also assign the priority to the patient supported their situation. It's useful when two ambulances arrive to the 
identical signal at same time. There is also certain decision that the software must take supported things of the signal lights. Variety of these possibilities is as follows if the signal is already green it'll remain the identical as long because the ambulance doesn't glide by. there's also a threat that individuals might think that it's technical error if it's green for too long only on one track and can break the rule to avoid this a greenhorn blue light is placed on the signal, whenever other signals be Red to grant way to emergency vehicle simultaneously the Blue light is lit as a sign that there's an emergency vehicle passing.

Dang et al [2] have proposed work which provides priority based approach. This aims to build an integrated user HPV

system through which an HPV driver can send requests to $t$ he system to which the system responds intelligently. Road Segments (RS) priority is determined at the intersection and light turns green with highest priority vehicle. They tested the algorithm on SUMO (Simulation of Urban Mobility) and it's shown positive results by saving over $50 \%$ time in various traffic intensities (low, moderate, high). The system mainly aims to tackle traffic jams problems for HPV. It's a user interactive system where the user (who is that the driver of HPV) initially attaches itself to the system before moving on for a call. It then sends an invitation to turn light green to the system at a traffic intersection to urge a green signal. The system calculates the priority of every RS of a traffic intersection. The system turns light green for the RS with highest priority value. The system takes good move by calculating the priority value of every intersection after a period of TLDC. Traffic Light Duration Cycle (TLDC) could be a time of 1 traffic cycle consisting of red and green duration of a light at an intersection. The model takes two scenarios into considerationSuppose there is no ambulance at a light inters ection on any RS then light runs automatically. Remember there are ambulances on every RS of traffic intersection as $t$ he system is determining the priority of any RS of a traffic $i$ ntersection then the RS with the best priority value turns gre en over other RS for a whole TLDC during this way the sys tem gives the priority to the ambulances and other essential vehicles.

Meera et al [3] explored about the opposite intelligent light systems and other transportation systems and came to conclusion and proposed this technique which has allow the android mobile device (emergency vehicle) to override the traditional operation of a stoplight. An android and cloudbased control system using the GSM module is an effective and price competitive solution that can solve this problem. The system comprises of 5 stages which are android mobile device, GSM module, MQTT (IoT) for Arduino IDE, Arduino Uno microcontroller and traffic signals. The developed system has allowed the android mobile device (emergency vehicle) to override the traditional operation of a stoplight. In this paper they developed an android and cloud based control system using the GSM module. The debates on mobility almost exclusively encompass functionalist analyses of how particular mobile technologies can alleviate geographical barriers for act. The developed system during this paper has allowed the Android mobile device (emergency vehicle) to override the traditional operation of a stoplight. They use an android and cloud based control system using the GSM module. The system comprises of Android mobile device, GSM module, MQTT (IoT) for Arduino IDE, Arduino Uno microcontroller and traffic signals this technique are going to be very essential for the benefit and safety of the people of the society, thereby ensuring that there's not a hold up that's experienced currently. MQTT is an "Internet of Things" connectivity protocol and is employed in sensors communicating in home automation and tiny device scenarios, which explains how the system works. The stoplight unit is going to be constructed and can be controlled by an Arduino Uno microcontroller with the relevant program code. . The android mobile device are going to receive messages and send commands to the microcontroller via the

cloud environment and MQTT cloud server with the GSM module is been used which may be compatible to both the cloud environment and therefore the Arduino microcontroller thus helps user to figure in user friendly mode.

Smith et al [4] have proposed the system which comprises of 5 stages which are Android mobile device, GSM module, MQTT (IoT) for Arduino IDE, Arduino Uno microcontroller and traffic signals this paper, they need proposed an adaptive Traffic Management System (TMS) combined with a symbolic logic based scheme so as to require appropriate actions to hurry up the progress of emergency vehicles while avoiding the creation of bottlenecks around their routes. This is often achieved through.The TMS has multiple steps at its disposal to ensur e the quickest possible response to an emergency; a number of these will be performed at dispatch time whereas others must be performed dynamically while the $\mathrm{EV}$ is on the way toward the emergency location.

Faldu et al [5] reported a paper on real time adaptive control system. In present world, the matter of traffic jam has become a significant concern. It's not just restricted to megacities or metropolitans but even for little cities; hence they require a wise or intelligent control system. Their present control system is not adaptive but relies on time and independent of the traffic density.

This static nature makes it noisy, unstable and inefficient. A true time traffic information processing and monitoring pro gram is proposed for addressing this problem of controlling traffic ppaths. This model monitors the clearance time of each lane sequentially and is based on the realtime density of traffic. The approach is very hybrid a mix of sensors networks and camera technology. A united result of image processing and IOT ensures more accuracy and efficiency instead of native approach. Besides traffic jam minimization, prioritization for emergency vehicle is additionally achieved through RFID. Other technology like stolen vehicle tracking/detection is additionally deployed. Simple GUI to be used adds a bonus to the system making it easy for room to handle. The model can be used for 
analysis purpose and hence predict traffic jams and traffic status at different times. Hence this model is cost-effective and time efficient smart control system. This model provides solution to the growing tie up problem and may effectively replace the present traditional methodologies or control system. Since the green path time could be a function of the traffic density it may be varied with the varying traffic densities and conditions. Clearance time calculation is adaptive and intelligent, additionally to the current prioritization for emergency vehicles and detection or tracking of the stolen vehicles makes it smarter. The hybrid approach employed in this model creates a grip over other methods. The info is collected at both local and central level hence makes it better for situation when one in all them fails. The cumulative density calculated gives precise clearance time since both the individual methods have some drawbacks in certain conditions that the hybrid model tries to scale back errors in one method and hence provide more accurate results. All the info collected and standing of the traffic is reported to the room and will be accessed by simple GUI. These may be further extended to indicate traffic status to the users. The info collected of traffic density at different times of day is used for analysis purpose and prediction of the traffic at different times of daily. The analyzed data then may be accustomed predict traffic jams at different locations of town. Hence the model could be a complete package for smart control system which may be extended for creating complete transportation intelligent.

Bhat et al [6] have done the analysis that the worst enemy of individuals living an urban lifestyle is commute. The exponential increase within the number of vehicles and transport options has led to an inevitable increase in congestion of pathways, which has necessitated the requirement for an efficient hold up control method. The system is aimed toward achieving control in developing countries like India, where additional parameters must be considered like road quality. It operates on the principle that at any instant of your time, no vehicle must be allowed to attend for longer than the typical vehicle waiting time, at that instant. It also prioritizes equipment such as ambulances, and recognizes unusual incidents such as ro ad accidents. The target of the system is to cut back the typical waiting time that every vehicle should wait, before it's allowed to pass, while also ensuring uniformity within the waiting times. The system is an efficient and highly economic solution to traffic problems in metropolitan cities in India, where exponentially increasing traffic may be a growing concern. The system is straight forward to implement, doesn't involve a good deal of complex computation, takes under consideration of parameters and almost accurately determines optimal average vehicle waiting time.

Punit et al [7] has presented the autonomic computing allude as a self-managing feature that autonomic system, manages the body. A system is autonomic if it can monitor changes by itself analyze, plan actions as per it and execute them automatically so as to become a reliable system. The current work focuses on a true life case study of light management system, plays a crucial role in our daily lives. In most places, especially in developing countries the light system is time bounded, which sometimes doesn't allow an ambulance carrying a patient to have light red light. Hence there should be a wise light signal system which may overcome with such problems, allow the ambulance to have stoplight whether it's red or green. This may be done employing a wireless sensor network and voice recognition technology. Implementation of this concept in traffic management by managing the traffic density on roads allows smooth and uniform drift of traffic. Making traffic signals autonomic, allows recognizing the wonted traffic density and voice of a specific vehicle and accordingly routes the traffic. Each approach has some advantage and disadvantage as during this approach it should be possible that voice recognition system may fail to acknowledge the ambulance siren thus identifying it as a standard vehicle or may sometimes recognize a normal vehicle as an ambulance, now days various forms of sounds are getting used as a horn sound on roads. Other factor which is defined as limitation, that if vehicles enfold only in sensor activated area vacating other expanse, then the signal might take it as inflated density of traffic and can decline the stand by time while there's less density. All of them knew that rules are for humans, but humans aren't for rules. Lifetime of an individual's being is far more important than following a rule which can result in worst situations. Thus, passing ambulance so, that it can reach the hospital as soon as possible and save lifetime of mortals. The long run scope includes the simulation of the approach using NS2, OMNeT++ or the other simulation software's and analyzing it for traffic management.

Janani et al [8] has done analysis study on road traffic jam becomes a serious issues for highly crowded metropolitan cities like, Chennai. Ambulance is one of the most critical

services affected by traffic congestion. This paper has come up with the solution of intelligent automatic control o $\mathrm{f}$ ambulance to smooth out the ambulance movement.Their program produces a cloud-based android app that links both the ambulance and the traffic light station. Design system makes uses oftenest Identification (RFID) technology to implement the intelligent traffic light control. The fundamental concept behind

the proposed system is to track the RFID tagged ambulance andsend the details to the cloud if the ambulance stops on $t$ he way due to a traffic light. After the user's acknowledgment via the mobile app, the actual signal is shaped green for many times and after the ambulance passes by, it regains its original signaling sequence flow If this scheme is compl etely

automated, the ambulance spot is identified, the traffic light $s$ are managed. Their system controls the traffic lights and save the time in emergency periods. It thus serves as a project for life savers. Human life is precious and must follow safety measures very conscious all told aspects 
this after all includes ambulances services too. In this, by using intelligent ambulance system they'll achieve the uninterrupted service of the control system by implementing the alternate methods for signal change to permit flow control. The accuracy of the RFID is quite camera's so this also improves the performance of stoplight violation detection system. Design system is cost effective, multiple usages and deployed using trending IOT, which is more efficient.

Singh et al [9] have done analysis study on tie up and tidal on current traffic management, which is facing two major problems in modern urban areas which cause road accident and loss of life. To overcome this, they introduced Automatic Ambulance Rescue System (AARS). The main idea behind this scheme is that by automatically monitoring traffic Lights on the route, ambulance can enter the hospital easily in time. The ambulance is operated by a control unit that provides the shortest route to the hospital and control traffic lights. The sensor senses the spot and also the nearest ambulance reaches the accident spot. The traffic lights within the path of the ambulance are controlled. The ambulance is driven along the shortest route to hospital by server. The vehicle unit installed in vehicle senses the accident and sends the placement of the accident to the server within the ambulance section. The server identifies the ambulance, closest to the spot of the accident and also the $\mathrm{s}$ hortest path between the ambulance, the spot of the accident and the nearest hospital. In this paper, they need described a design for automatically controlling the traffic signals so the ambulance would be ready to cross all the traffic junctions and reach hospital without time delay. Human life is affected to risk by the delay within the arrival of ambulance. The ambulance isn't ready to reach the hospital within the golden hour. The prevailing system has many disadvantages. It depends on the way of monitoring people to be manual which ends in time delay and since of that health services can not be provided to the patient on time which results in loss of human life. The ambulance is guided to the hospital by the central unit through the shortest route. The sensor installed within the vehicle senses the accident and Global Positioning System (GPS) tracks the placement of the accident. It sends the incident location

the emergency section via Global System for Mobile Comm unications (GSM). The central unit finds the ambulance, nearest to the accident spot and also the shortest path between the placement of the accident, ambulance and therefore the nearest hospital. Here, wireless technologies are accustomed transfer information.

Bhilawade et al [10] has done analysis study on over the globe, there has been a rapid increase in vehicle numbers. There are approximately 1 million licensed vehicles within the last year so that traffic problems has increased within the previous couple of years and therefore the present light controllers have limitations because it uses the fixed hardware which do not have the flexibleness of modification on real time basis. Therefore the time intervals of green, orange and red signal $\mathrm{s}$ are set, so the waiting time is greater. To form this light controlling more efficient they emerge new technique called as intelligent control system. This uses sensors together with embedded technology. The timings of the red and green lights are smartly decided supported the traffic on roads. As compared to previous fixed mode light controller this new system is more efficient and flexible. It also has facility to pass the emergency vehicles like ambulance, fire brigade etc. so detecting and also recording stolen cars. The look also has scope for further expansion. Green wave system was accustomed provide clearance to any emergency vehicle by turning all the red lights to green on the trail of the emergency vehicle, the largest disadvantage For the green waves, the disruption will cause traffic problems when would be intensified by the synchronization. In such cases, the queue of vehicles during a green wave grows in size until it becomes overlarge and a few of the vehicles cannot reach the green lights this is called over abundance in time, and must avoid.

\section{CONCLUSION}

The existing system doesn't provide a transparent path for emergency vehicles during traffic congestion. From the literature survey, we've found that RF ID-based smart traffic control system provides an answer to the traffic congestion problem and this can be also an efficient method to supply a transparent path for the emergency vehicles when identified within the lane, as we also implemented sharing of patient's vital data with hospital we updated Arduino uno with Arduino mega board so it'd be sufficient for storing of patient vital parameter and simultaneously it performs capturing of present status of traffic signal present in different path and we also added another system in the junction which repeatedly scans the density of the lanes so that the system can automatically allow the lane which has high density by this technique the emergency vehicles experience less congestion and reach faster to the destination and thus many life's were been saved.

\section{REFERENCES}

[1] R.S.B.Vanjale, SayaleeDeshmukh."IOT based Traffic Signal control for reducing time delay of an Emergency Vehicle using GPS", April 6-8, 2016, India. Issue 5page, IEEE ISBN:978-15090-0396-9/16/\$31.00 @2016 IEEE.

[2] Dheeraj Dang, JitinTanwary and SarfarazMasoodz."A Smart Traffic Solution for High Priority Vehicles", 4-5 September 2015 ,ISBN: 978-1-4673-6809-4/15/\$31.00 @2015 IEEE.

[3] Meera K, Mpho K. Madisal. "Android and Cloud based Traffic Control System”, ISBN:978-1-5386-3060- 0/18/\$31.00 (C2018 IEEE.

[4] Nicolas Smith,SoufieneDjahel, Shen Wang, and John Murphy. "Reducing Emergency Services Response Time in Smart Cities: An Advanced Adaptive and Fuzzy Approach",ISBN:978-1-46736552-9/15/\$31.00@ 2015IEEE.

[5] Prayushi faldu,Nishath Doshi."'Real Time Adaptive Traffic Control System" ISBN: 978-1-7281-1322-7/19/\$31.00 (C2019 IEEE.

[6] Amit Bhat, Kaushik Roy, Prajesh P Anchalia, and Jeevith HM. "Design and Implementation of a Dynamic Intelligent Traffic Control System"| ISBN:978-1-4799- 8713-9/15 \$31.00@ 2015 IEEE DOI 10.1109/UKSim.2015.12. 
[7] Puneet Kumar Aggarwal,Prashanth nigam,Vineeth shrivastava."Self Controlled Traffic Management Using Autonomic System", Issue page 3, ISBN:978-9-3805- 44212/16/\$31.00 @2016 IEEE 2016.

[8] B.Janani Saradha,G.Vijayshri,T.Shubha."Intelligent Traffic Signal Control System For Ambulance Using RFID" And CLOUDs: ISBN: 978-1-5090-6221-8/17/\$31.00@2017 IEEE.
[9] Smriti Singh, Tandrima Chowdhury, Maflin Shaby. " A Rescue System of an Advanced Ambulance Using Prioritized Traffic Switching", ISBN: 978-1-4799-6818-3/15/\$31.00 @ 2015 IEEE.

[10] Vidya Bhilawade, L. K. Ragha.'Intelligent TrafficControl System", Volume 8, Issue 2, February 2018. ISSN 2250-3153. 